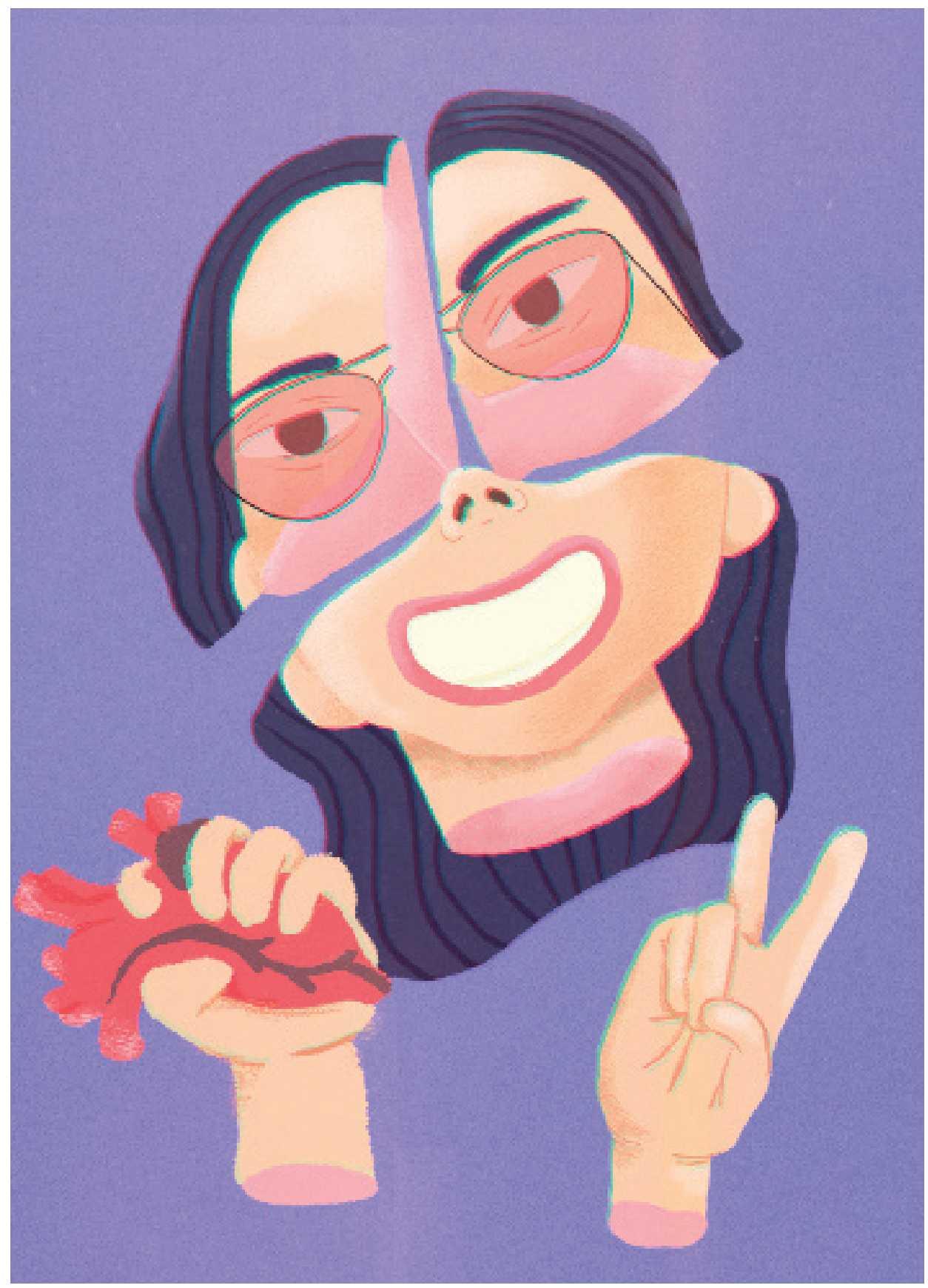




\title{
Lo cotidiano de las partes del cuerpo y su vida social
}

\author{
Entrevista a \\ Raquel Díaz Bustamante ${ }^{1}$
}

\author{
Por: \\ Ana Lucía Cortés
}

Universidad Icesi

analuciacortesghgh@gmail.com

Entrevista recibida 12/06/2019

y aprobada $24 / 07 / 2019$.

Cómo citar este artículo:

Díaz, R., \& Cortés, A. L. (2019). Lo cotidiano de las partes del cuerpo y su vida social. Trans-Pasando Fronteras, (14). https://doi.org/10.18046/retf.i14.3652

1 Antropóloga, Magíster en Bioética, Pontificia Universidad Javeriana Bogotá. Correo de contacto: raquel.diaz@correo.icesi.edu.co 
Raquel Díaz Bustamante es antropóloga de la Pontificia Universidad Javeriana de Bogotá. Durante su formación universitaria participó en el énfasis de antropología de la salud y esto la llevó a hacer una maestría en Bioética en la misma institución. Desde hace varios años ha trabajado como docente en la Universidad Icesi y ha podido investigar sobre el tema del cuerpo en temas concernientes a la salud sexual reproductiva, la donación de órganos y tejidos y el uso de partes anatómicas en el desarrollo de tecnologías médicas y medicamentos biotecnológicos.

El tema de la donación de órganos es uno del que no se habla todos los días, sin embargo, es tan cotidiano como la vida misma, ya que se refiere a nuestro cuerpo, a la salud y también a la muerte. En el año 2016 se dio a conocer la Ley 1805 del 04 de agosto, cuyo propósito es el de ampliar la presunción legal de donación de órganos y tejidos humanos para trasplantes y otros usos terapéuticos (Ley 1805, 2016).

Durante el mismo año se llevó a cabo un proyecto de investigación que se vio bien favorecido en un concurso de inter-facultades en la Universidad Icesi, logrando un acercamiento entre las facultades de derecho y ciencias sociales y la de medicina. Este proyecto se hizo con el aval de la unidad de trasplantes de la Fundación Valle del Lili. El proyecto se realizó durante el año 2016, de enero a diciembre y no tuvo continuidad.

El trabajo de campo realizado en el marco de este proyecto de investigación tuvo lugar dos meses antes de que se aprobara la Ley 1805, lo que hizo que las respuestas de las personas fueran por la vía de la expectativa, aunque realmente muy pocas personas conocían y sabían en qué consistía este nuevo proyecto de ley, lo que da cuenta de la poca difusión que se dio con respecto a ésta. Dicho proyecto de investigación indagó sobre los aspectos 
socioculturales y los dilemas éticos que inciden en la disposición de pacientes, usuarios y cuidadores de la Fundación Valle del Lili. Varios de los aspectos indagados permiten reflexionar sobre la vida social de los órganos y los tejidos de los seres humanos.

\section{A: ¿A qué hace referencia la metáfora de la vida social de los órganos y tejidos humanos?}

R: La vida social de los órganos es una metáfora acuñada por las antropólogas Margaret Lock y Vinh- Kim Nguyen, la cual hace referencia a cómo le damos un sentido, una biografía, un significado o un valor simbólico al cuerpo y sus partes. La vida social de los órganos habla de cómo nosotros conocemos y fragmentamos el cuerpo sin que esto necesariamente esté del todo mediado por el discurso médico, se refiere a cómo la gente comprende las cosas que le sucede a su corporalidad en lo habitual. Es una metáfora que permite pensar en aspectos socioculturales que se construyen en lo cotidiano y que muchas veces son invisibles. Algo muy común es que uno solo se da cuenta que tiene un estómago cuando se enferma, cuando tiene gastritis o una gastroenteritis; uno se da cuenta de que tiene un cerebro cuando, por ejemplo, siente estrés y le duele la cabeza. Pero también hemos asignado significados a ciertas partes por lo que aluden a las emociones, como pasa con el corazón.

Nosotros nunca nos estamos cuestionado "¿dónde está la tiroides? o ¿dónde está el apéndice?”, sin embargo, hay manera en las que estas se manifiestan y la gente construye un significado, un valor a éstas. Entonces la vida social de los cuerpos en el contexto de donación de órganos y tejidos consiste en darle un sentido a una parte que sale de un cuerpo y que entra a otro, al de un donante, persona receptora de este siempre tiene preguntas 
sobre esta nueva parte y no siempre obtiene respuestas sobre la historia de vida de dicho componente anatómico.

\section{A: ¿Cómo se pueden abordar metodológicamente estas re- laciones entre humanos y no humanos o partes humanas? ¿Cuál sería un punto de partida, por ejemplo?}

R: Un punto de partida es volviendo a lo cotidiano. Y eso es como un desafío del investigador o del etnógrafo. El punto de partida es el propio cuerpo del investigador, entonces uno desde sus propias experiencias corporales va a preguntándose y va pensando cómo hacer que las otras personas puedan construir respuestas sobre esto. Yo creo que uno se hace consciente de su cuerpo en la salud y en la enfermedad, cuando bajas mucho de peso y te sientes bien, o cuando está enfermo. Pero también hay otras formas y eso se ve a partir de oficios, de prácticas, por ejemplo, uno se hace consciente de que tiene un manguito rotador cuando en el oficio, sobre todo oficios domésticos que te exigen fuerza o en los trabajos manuales. Uno de mis referentes es mi mamá, ella es diseñadora de modas y hay días en los que le duelen mucho las muñecas, el codo, y es porque la postura de sus manos y brazos le produce esto. Yo sólo soy consciente de mi codo cuando me pego durísimo, sin embargo, por su oficio y por su quehacer, ella sabe cuándo se le va a manifestar esa parte del cuerpo que normalmente no es considerada como algo muy presente en su vida. Lo mismo le debe de pasar, yo creo, a millones de personas bajo muchísimas circunstancias, entonces son esas prácticas del hacer cotidiano, los detonantes que un investigador puede tener como punto de partida, osea, lo cotidiano, sus relaciones con otras personas y con su propio cuerpo. 


\section{A: ¿Cómo le parece que es trabajar este tipo de temas co- tidianos en Colombia?}

R: Desde la antropología médica crítica, yo creo que uno puede hacer lecturas de este tipo. A través de las experiencias que tienen las personas con sus cuerpos, con la salud o la enfermedad, uno puede indagar sobre la vida y la cotidianidad de las personas en términos de la desigualdad social que se vive a la hora de acceder a los servicios de salud, puede hablar de género y cuestiones sobre la salud sexual y reproductiva de hombres y mujeres; uno puede también indagar sobre lo político o de lo biopolítico, como esas cuestiones de bueno, este es mi cuerpo, yo me hago cargo de éste, yo lo cuido, lo nutro, pero igual también hago parte de un proyecto político más grande y un ejemplo de eso es la Ley 1805 de 2016 que habla o dice que todos los colombianos somos donantes y si me niego a la donación, a lo que dice este proyecto, no pudo ser candidato a un trasplante. Lo mismo pasa con temas como la eutanasia o la interrupción voluntaria al embarazo, esas cuestiones nos hacen incluso cuestionar esos alcances que nosotros tenemos para gobernar nuestro propio cuerpo y cumplir con el deber ciudadano.

\section{A: ¿Cómo es trabajar estos temas desde las ciencias sociales?}

R: Aunque la antropología médica crítica es un campo en expansión, debo reconocer que a veces investigar sobre estos temas es muy difícil, porque en ocasiones uno no encuentra mucha bibliografía sobre ciertos temas, entonces en ese sentido, trabajando estos temas del cuerpo, sobre salud, sobre tecnología médica, me he obligado a ser muy indisciplinada o "inter-disciplinada". A la hora de leer artículos científicos y relacionarme con médicos, biólogos, microbiólogos, entre otros, me ha salvado la vida haber 
hecho cinco semestres de biología, pero también me ha tocado aprender sobre epidemiología, la salud pública, la psicología, de la psiquiatría, de los estudios sociales en ciencia y tecnología. Porque todos estos campos tienen mucho que decir sobre el cuerpo, sus partes, pero, sobre todo, las prácticas que realizan los seres humanos a través de este y su sentido social. Cada vez más emergen nuevas miradas para pensar el cuerpo desde las ciencias sociales, sin embargo, creo que uno no debe de desconocer y hacer de lado los discursos de las ciencias naturales y de la salud para, por ejemplo, poder investigar sobre la donación de órganos y tejidos, el cual es un tema de importancia social muy grande y para abordarlo como científicos sociales, un buen punto de partida es indagar desde ese discurso, el biomédico, el que siempre se ha dado y darle la vuelta de manera etnográfica a esos temas y ahondar sobre las experiencias corporales que hacen alusión a la intención de donar o no de manera cualitativa; desde las respuestas de la gente a los por qué, para qué y cuándo.

Entonces este ha sido mi punto de partida y es la manera en la que comienzo a fabricar mis reflexiones sociales, políticas, bioéticas sobre estas cuestiones, entonces sí, las ciencias sociales es un campo muy amplio que nos permite tener herramientas, pero también es cierto que otras disciplinas aportan a la comprensión de estas prácticas, de estos significados, entre otras cuestiones.

\section{A: ¿Cómo es ese trabajo interdisciplinar o multidisciplinar, por ejemplo, trabajar con personal de salud o de otras disciplinas?}

R: Es complicado, sobre todo porque nosotros como antropólogos, sociólogos, politólogos, queremos construir ciencia y hacer ciencia, pero la versión o el significado de ciencia que 
se tiene sobretodo en el campo de la salud o de las ciencias naturales, es objetivo, es numérico, siempre se basa en evidencia y en datos obtenidos a través de los pasos del método científico. Y lo que pasa con nosotros en las ciencias sociales es que nuestros hallazgos dan cuenta de muchas cuestiones o reflexiones que no siempre son verdades absolutas; habla de subjetividades, de reflexiones, de críticas y "Ios datos" no tienen que ser objetivos, sino más bien respuestas que nos lleven a reflexionar de manera crítica sobre el porqué no nos gusta donar órganos y tejidos, aunque nos parece un acto bondadoso y admirable. Y lo cierto es que, a mí como investigadora social, las encuestas sobre este tema en particular no me explican el por qué del asunto, pero a los médicos con los que trabajamos sí les parece que es la forma correcta para seguir explorando esta problemática.

Entonces digamos que trabajar con estas otras disciplinas no es muy fácil, hay que colmarse de paciencia, pero sobre todo, siempre debemos hacer el ejercicio de traducción, de cómo nosotros aportamos a lo que ellos hacen. Muchas veces esos ejercicios de traducción no son muy fáciles, hay quienes están 'parados en la raya' de que no, lo cualitativo y lo cuantitativo deben ser totalmente opuestos; en mi caso, yo he aprendido que deben de ir juntos, la investigación mixta permite la traducción y un intercambio lingüístico entre lo numérico y lo narrativo; se complementan muy bien porque muchas veces las cifras no me responden los por qué, el cómo y el para qué, entre otras cuestiones y es en esas preguntas donde yo encuentro los significados de aquello que es habitual y de lo que no siempre estoy reflexionando cotidianamente.

Tengo la sensación que hay muchas cosas que damos por sentado sobre nuestra forma de relacionarnos, de disponer del cuerpo y sus partes, no sé, si hacemos una encuesta sobre si usted 
se lava las manos cada vez que va al baño, probablemente un porcentaje alto de personas va a decir que sí y otro va a decir que no; pero la verdad es que dudo que ese gran porcentaje de personas que dicen sí lo hagan siempre. También me quedaría la gran curiosidad por entender el porqué las personas valientes que dijeron 'no', han dejado de lavarse las manos en varias circunstancias. Son esos por qué y para qué los que me hablan también de esas actividades muy cotidianas que estamos dando por sentadas. Entonces me parece que lo 'cuali' y lo 'cuanti' se complementan muy bien porque permiten visibilizar cuestiones que a uno a veces no se le ocurren, no nos podemos quedar solo con el dato numérico, también es necesario lo narrativo.

\section{A: Concretamente, ¿cómo se aborda el tema de la donación de órganos y tejidos desde las ciencias sociales?}

R: En antropología médica crítica se está haciendo a nivel de la mercantilización y fragmentación del cuerpo, digamos que en Colombia esto es una preocupación muy grande porque la gente siempre tiene temor de que las partes de su cuerpo van a ser vendidas, van a ser comercializadas, que se les va a perseguir, los van a asesinar, y yo creo que eso no viene de gratis, es del contexto en el que vivimos. Esas inseguridades, esos miedos y esas violencias que nos rodean también se permean en este caso, entonces hay mucho cuestionamiento en torno a los procesos de donación de órganos y tejidos y, sobre todo, qué tan transparentes son en nuestro sistema de salud. Otras cuestiones que emergen alrededor del tema y sobre las cuales las personas conversan mucho, son obviamente los significados más subjetivos, el valor simbólico o esa vida social de los órganos y tejidos que hablan sobre prácticas de autocuidado y también de lo que la persona en- 
tiende por qué es un trasplante o qué es una donación, qué es un órgano y un tejido. Otra manera de abordar este tema es a través de la compresión de la política pública o de la ley 1805, es decir, qué implicaciones tiene eso para la vida de un individuo que no tiene intenciones de donar. Sin embargo, desde que aprobaron esta Ley, no se ha profundizado mucho sobre el tema, tampoco ha habido una socialización sobre esta; yo creo que la invitación también es a empezar a entender cómo la gente está entendiendo estos procesos biopolíticos que nos llevan a ser donantes en Colombia y eso todavía yo no lo he visto.

\section{A: ¿Cómo se construyen metodologías para abordar las re- laciones entre lo humano y lo no humano para este caso es- pecífico? ¿En este caso cuál puede ser un punto de partida?}

R: Nuestros puntos de partida fueron nuestros miedos y nuestros dilemas frente al tema. Adriana Granados Barco fue mi compañera en todo esto, ella es trabajadora social y tiene una sensibilidad hacia lo cualitativo muy bonita, pero entonces lo que hicimos, nuestro punto de partida fue ponernos en los zapatos de ciudadanos y ciudadanas que van a ser donantes. Nosotras por ejemplo descubrimos que sí, que ambas queríamos ser donantes, pero nunca habíamos realmente conocido cómo y en qué consistía esta práctica médica. Entonces, al comienzo de la investigación, sí se nos hizo un poco violento eso de sacar órganos y tejidos. Sin duda es una práctica que al ser descrita por los médicos suena violenta, atemoriza y me hace pensar que mi cuerpo no va a ser el mismo; entonces pasamos a preguntarnos sobre las cuestiones estéticas, sobre la apariencia del cadáver, y eso, también nos permitió tomar decisiones políticas sobre nuestros cuerpos, nos hizo pensar en que nosotros tenemos que 
socializar nuestra intención de donar con nuestras familias. Tras estas reflexiones, lo primero que hicimos fue hablar con nuestras familias, comentarles acerca del proyecto de investigación y la Ley 1805 que aún no había sido aprobado, con el fin de conocer su perspectiva, qué opinaban al respecto. En nuestros casos nuestros familiares son muy conscientes que en el momento en el que toque tomar la decisión, se va a optar por la donación y eso es una respuesta consensuada que se respeta. Pero entonces eso también nos sirvió para comenzar a pensar críticamente sobre cómo son esos procesos en otras familias, cómo es la concepción del cuerpo que se tiene, pues al tener en mente la metáfora de la vida social de órganos y tejidos, cada sujeto fragmenta y le da un significado a los ojos, la cara, la rodilla, el hígado, el riñón. Pero resulta que descubrimos que dicha fragmentación del cuerpo en cada parte sirve para entender la importancia que tiene para un individuo poseer y morir con un cuerpo completo.

Realmente una de los dilemas éticos que encontramos entre los participantes de la investigación, fue que las personas consideran que su cuerpo debe de estar completo para ir al más allá, ya sea desde una visión judeocristiana o de una no religiosa; todas y todos comparte que si vienes completo te tienes que ir completo. También comenzamos a entender razones sobre laestéticas del cadáver, para nosotros como colombianos y colombianas es muy importante tener un ataúd abierto en el rito fúnebre y uno ve cómo las personas van a observar "al muerto" en medio del funeral, ya sea por morbo, por respeto, por fascinación, pero digamos que el cadáver, la presencia del cuerpo que fallece, es muy importante para hacer cuestiones de duelo. Eso entonces también nos llevó a preguntarle a estas personas cuál es la importancia del cuerpo en ese duelo, que lo habíamos pensado solo por la vía de bueno, uno muere, se convierte en materia orgánica o uno termina reducido a cenizas, pero también 
para las personas entrevistadas importa esa parte estética, de que te veas sonriente o dormido dentro de un ataúd. Entonces digamos que nos tocó hacer un ejercicio de mucha empatía, preguntándonos incluso sobre nuestra propia muerte y qué responsabilidades políticas tenemos sobre nuestro cuerpo y qué derechos tenemos. Y sin duda hay una categoría importante para entender la manera de disponer de nuestro cuerpo en el momento de nuestra muerte y es el de la familia donantes. Las familias donantes son aquellas que tienen un paciente en estado crítico y es candidato a una donación; esta situación hace que este grupo familiar afronte cuestiones y situaciones muy particulares sobre la muerte.

En los grupos focales o en los espacios de participación ciudadana (EPC) que realizamos en el marco de esta investigación, porque realmente nosotras tuvimos que llegar a este punto de plantearnos una metodología o espacio, el tema de la muerte emerge como un tabú o un elemento que no puede ser nombrado o pensado, porque los pensamientos la atraen. Los criterios de selección de los participantes de los cinco espacios de participación ciudadana fueron el de estar entre los 18 y los 65 años (la población se segmentó en EPC de 18 a 25, de 25 a 45 y de 45 a 65 años en adelante), estar afiliados al sistema de salud ya sea en condición de régimen subsidiado o contributivo, y por encargo institucional, haber asistido a la Fundación Valle del Lili en calidad de paciente, usuario o cuidador. Hablar sobre la muerte y el manejo del cadáver fue el hilo conductor de los debates que estos espacios propiciaron.

Esta metodología permitió que los y las participantes deliberaran sobre el tema de manera libre, compartiendo preguntas, inquietudes y sobre todo, sus vivencias en torno al tema de la muerte y la donación; fue así como descubrimos que varias de 
estas personas eran personas trasplantadas o que fueron familias donantes. Pero nos Ilama mucho la atención que el resto de las personas que no han tenido experiencias cercanas a esto o le dicen que un no rotundo a la donación, creen que hablar sobre la donación de órganos y tejidos es invocar a la muerte y es de mala suerte. Sin importar si tienes 25 años, estás en el Sisbén, en una prepagada o si estás en una EPS, la gente no quiere hablar de del tema de la donación porque evoca a la muerte y sin duda eso fue un hallazgo muy grande y metodológicamente, fue un reto. Esto nos obligó a que en cada EPC se aplicaran tres técnicas: un taller de mapeo corporal que hablara de esa vida social de los órganos y tejidos, un grupo focal que les permitiera a estas personas compartir aspectos socioculturales que vincularán creencias, costumbres, prácticas, rituales entre otros, a la manera en la que estas personas viven en su cuerpo.

Pero además, dicho grupo focal permitió discutir y debatir cuestiones más administrativas del proceso de la donación y fue ahí, sin duda, cuando emerge la variable del miedo a la mercantilización del cuerpo y sus partes: muchos hablaban de la existencia de un mercado negro, sobre el tráfico de órganos en instituciones médicas y sus dudas o total desinformación sobre la Ley 1805. La última parte de los EPC, fue la reproducción de un video en el que se muestra cómo se hace el rescate de un órgano en un hospital o en una clínica y uno de los requisitos que deben asumir las familias donantes, es el de enfrentarte a una entrevista con coordinadores de una unidad de trasplante, que te piden si quieres donar los órganos y tejidos de un familiar cercano, un amigo, un conyugue, entre otros.

Entonces las situaciones que recreó este video permitieron que la gente hiciera comentarios bastante sinceros en términos 
emocionales. Al mostrar este video queríamos que la gente también manifestara su tristeza, rabia entre otras emociones y creo que lo logramos. Nunca fue nuestra intención hacerlos Ilorar o entrar en estado de cólera, pero también quisimos correr el riesgo para así hablar sobre los dilemas éticos que ellos y ellas imaginan enfrentar en el momento en que decidan por ellos o por un tercero frente a la donación.

Incluso, nos enfrentamos a situaciones de mucha tristeza cuando algunos participantes narraron cómo se enfrentaron a esta situación al negarse ante donar un órgano o tejido y cómo se arrepintieron después. Este, el de arriesgarse metodológicamente, ha sido un ejercicio de entender la magnitud del manejo de las emociones que encierra un tema tan cotidiano como la muerte y la donación de órganos y tejidos; esto no tema que debería de ser un tabú, ni muchos debería estar relegado al momento en el que toque decidir, esto es de esas decisiones biopolíticas que todo ciudadano debería tener siempre presente, incluso, desde el colegio, desde que estás en kínder, cuando construyes una relación con tus padres, en pleno proceso de socialización con tu cuerpo. Esto me ha llevado a pensar que te deberían de explicar que la muerte hace parte de la vida y que sin duda también toca pensar y tomar decisiones en torno a eso.

Esto significa invocar o atraer la mala suerte, pero, sin embargo, lo que arrojó esta investigación es que sí, donar órganos y tejidos para la mayoría de personas tiene una carga moral y simbólica muy fuerte, entonces digamos que todas esas cosas nos hicieron mucho ruido y nos llevaron a construir una investigación con estas características y no fue fácil, muchas veces después de cada grupo focal, de ver el tipo de emociones y de historias de vida que se narraban ahí, nosotras llegábamos car- 
gadas y emocionalmente muy inquietas, sobretodo porque por qué nos cuesta trabajo hablar de algo tan habitual como lo es el cuerpo y como lo es la muerte.

\section{A: ¿Cuáles son esas otras metáforas que permiten hablar de estos temas?}

R: Una emergente en la investigación es la economía de la salvación, esa categoría a mí se me metió entre ceja y ceja porque sí habla de una serie de intercambios que se tienen que hacer, no sólo corporales sino también emocionales, simbólicos, para que algo como un trasplante, una donación se haga efectiva. Otra categoría u otra metáfora o frase que me llama la atención es "si completo vine al mundo, completo me tengo que ir", y esa frase fue muy recurrente entre las madres que participaron en los grupos focales; significa algo como yo le di vida a este ser humano y salió completo de mi vientre, por qué yo tengo que descuartizarlo. Descuartizar es una categoría emergente que habla de cómo la gente entiende la práctica como algo simbólicamente muy violento y eso nos llevó a una reflexión sobre la violencia simbólica que hay detrás de esta práctica.

Otra categoría fue la de esperanza de vida, entonces digamos que esta categoría emerge cuando se hablaban o se contaban casos de donación de órganos y tejidos de personas muy jóvenes o de niños, ya fuese propias, vistas en la televisión o vividas por personas cercanas a ellos. Un cuerpo humano permite salvar cincuenta y dos vidas, pero incluso esos son cálculos utilitaristas, realmente uno puede salvar incluso más vidas porque las personas que rodean ya sean sus familiares o cuidadores, también se salvan, porque cuando uno está enfermo ese es un evento que afecta a cuidadores, y por cuidadores me refiero a mamá, papá, 
una tía, un primo, o una persona externa. Entonces, digamos que esas han sido las categorías emergentes que más me han Ilamado la atención, de pronto sí podemos llamarlas metáforas, y eso es muy bonito porque muestra la importancia de la narrativa. Como esta investigación fue mixta, las 300 encuestas realizadas en los pasillos de la Fundación Valle del arrojaron que el 91\% de las personas encuestadas tenían la intención de donar órganos y tejidos. Sin embargo, cuando nosotros hicimos la parte cualitativa, descubrimos que la gente tiene muchos temores y muchas desconfianzas, temen que las partes de su cuerpo que son valiosas terminen en cuerpos de personas malas y entonces aquí digamos que emerge el tema del conflicto armado y de la violencia. La gente decía cosas como "yo no quiero que me saquen partes de mi cuerpo y terminen en el cuerpo de un guerrillero o de un desmovilizado o de un paramilitar, tampoco quiero que a mí me done partes del cuerpo una persona que fue mala, un sicario, imagínese".

Estas personas creen que cuestiones como la ética se transmite a través de una parte del cuerpo, entonces encontramos que de los cincuenta y ocho participantes de los EPC la gran mayoría cree que, por más que tengan la intención de donar, hacerlo es muy difícil, no, incluso, mucho más de la mitad, casi todos creen que hacerlo es muy difícil, es muy fácil enunciarlo pero hay muchas variables que les impide realmente hacerlo, ya sea por la esa vida social que construyen sobre su cuerpo y sus partes, o por cuestiones sobre propiedad del cuerpo que incluso ni siquiera van por la mirada del estado, porque quiénes pueden tomar decisiones sobre mi cuerpo cuando las personas a mi alrededor están en situación de familias donantes, puede ser una tía que yo no veo desde hace tres años, mi papá que nunca convivió conmigo, entre otros. Las personas alrededor del paciente en estado crítico o de muerte cerebral siempre van a opinar. Entonces diga- 
mos que es un tema que se complejiza a través del duelo. Cada vez que vuelvo a hablar sobre esta experiencia y vuelvo como a reflexionar sobre los retos metodológicos que afrontamos, me convenzo de que antes de morir debo tomar la decisión si voy a donar o no y no dejar que terceros, que afrontan una tristeza muy profunda y un duelo, lo hagan.

\section{A: ¿Cómo se construye la identidad social de los órganos?}

R: Yo creo que eso se construye a lo largo de una historia de vida, de una vivencia, de cada cicatriz, cada vez que te da una gastroenteritis, tras hacerte un nuevo tatuaje, te cortas el pelo de cierta manera. Creo que uno le va dando sentido a esa vida social día a día, en mi cotidianidad, yo construyo una biografía sobre cada parte, lo que pasa es que no somos conscientes de esa fragmentación que hacemos a través de estas prácticas, por ejemplo, cuando las personas van al gimnasio, entonces uno le pregunta a una mujer: "bueno, ¿por qué vas a el gimnasio?" y responden "no, es que no tengo cola". Si le pregunta un hombre dirá: "quiero tener una chocolatina marcada en el abdomen". Uno podría decir, ah, pero eso es por vanidad.

Pero si lo pienso con detenimiento, uno puede llegar a preguntar: ¿Qué sentido y qué valor social le das a esta parte del cuerpo? ¿por qué es tan importante la apariencia de estar partes del cuerpo? ¿Quién me lo enseñó o cómo me di cuenta de esto? ¿Por qué son tan importantes los glúteos y los abdominales? ¿Cuál es la vida social de estas partes del cuerpo y la persona que las posee y desea mejorar? Tanto hombres como mujeres tienen mucho que decir al respecto y eso puede tener un punto de partida en esa biografía social de los órganos y tejidos, que dicen cómo se 
piensa y vive en un cuerpo y que está entrelazado en término de costumbres, imaginarios, mitos, hábitos, entre otros, que sin duda por esa socialización que uno tiene con las otras personas y otros cuerpos, se aprehenden.

Entonces digamos que la vida social de los órganos y tejidos es una cosa que me permite construir un puente entre las prácticas biomédicas y con las prácticas cotidianas de las personas, sobre temas que siempre están presentes en nuestras conversaciones, en nuestras formas de vivir y pensar, pero que nunca pasan por esa reflexión crítica de por qué las enuncio y por qué esto le da sentido a la forma en que yo habito mi cuerpo.

\section{A: ¿Qué piensas al respecto de cómo socializar la ley 1805 con otras comunidades étnicas y culturales en Colombia que conciben el cuerpo de múltiples formas?}

R: Es que esto es necesario, y ahí sí como antropóloga pienso que si nosotros hemos permitido, facilitado, traducido prácticas, diversas formas de lenguaje, rituales, entre otros para facilitar la construcción de proyectos políticos o políticas públicas, pensando la variable étnica, entre muchas otras, pues también toca socializar este tema con absolutamente todas las formas de vivir y ser en este país. Una vez hice un taller sobre la vida social de los órganos y un estudiante perteneciente a una comunidad afro me dijo "no, es que bajo mis creencias y mis prácticas rituales mortuorias yo no puedo donar una parte del cuerpo, es algo esencial para mi tránsito de la vida a la muerte y lo que sigue después" y yo lo que siento es que esa ley 1805 de 2016 fue aprobada en silencio, nunca fue socializada, por más de que la W, o al final de esas novelas que hablan sobre tráfico de órganos se toque el tema, 
este proyecto de ley deja por fueras esas otras formas de morir y pensar la vida social del cuerpo y sus partes en el más allá.

No existen espacios de participación ciudadana que permitan pensar y construir una posición al respecto. Este fue el mayor aprendizaje de este proyecto, darle la palabra a la gente y preguntarles como les gustaría aprender sobre la donación de órganos y tejidos para saber si hacen el trámite en la EPS a favor del sí o del no. Entre esas formas de cómo hablar del tema, las personas nos decían: "vayan a un centro comercial y le cuentan a la gente, pónganle un video, hagan un documental"; Netflix es una plataforma por la cual la gente se está enterando de muchas de estas cosas. Sin embargo, yo como profesora de bioética me he visto en la obligación de tocar el tema en mis clases y ya si sorprenderme me doy cuenta que nadie ha leído el PDF de la Ley 1805 y eso es preocupante; si en contextos urbanos encontramos un montón de aspectos socioculturales que no le permite a la gente donar órganos y tejidos, imagínese cómo será abordar el tema en contextos donde lo étnico, lo rural, lo campesino, tiene unas lógicas de entender el cuerpo muy particulares, a través de rituales, de prácticas, que siempre hablan de la salud, de la enfermedad de otra forma y que tienen la misma relevancia.

Desafortunadamente este proyecto de investigación sólo fue pensado en términos urbanos porque teníamos el aval de una institución médica, pero sí me hubiese gustado tener más variedad y si en algún momento se me da la oportunidad, me gustaría indagar sobre esas otras formas de entender estos procesos. Si para la gente en contextos urbanos la donación de órganos y tejidos es una cosa tan violenta en términos simbólicos, pues entonces imagine el impacto que esto tendrá en otras cosmovisiones; hasta de pronto llega a ser incluso más violento. Sí, la Ley 1805 
es un proyecto que puede salvar vidas y probablemente se construyó con la mejor intención, pero hasta que todos los ciudadanos y ciudadanas colombianos y colombianas se enteren bien de qué es esto, a mí me parece que en sí es una forma muy complicada y hasta grosera de fomentar esta práctica, osea, necesitamos que el número de donantes suba, pero ¿es esta es la mejor manera? Por qué siempre la construcción de este tipo de proyectos políticos no considera la mirada del ciudadano y de la ciudadana, la diversidad étnica o los aspectos socioculturales de las personas que viven en ciudades. Realmente vivimos en un país marcado de muchas desigualdades sociales y políticas, pero también hay una diversidad de entender cómo uno dispone de su cuerpo. Entonces la invitación es a construir espacios de socialización de esta nueva ley, que la ley 1805 de 2016 no se quede en un PDF y se construyan más formatos no sólo para hablar de esto, sino para todas las prácticas médicas que nos conciernen a todas y todos como ciudadanos. 